\title{
ATLAS status, operation and performance
}

\section{Lidia Smirnova ${ }^{1}$}

\section{on behalf of the ATLAS Collaboration}

D.V.Skobeltsyn Institute of Nuclear Physics, Moscow State University

Leninskie Gory 1, 119991, Moscow, Russian Federation

E-mail: Lidia.Smirnova@cern.ch

\section{Abstract}

In 2010 detector ATLAS was completely functional with $3.5 \mathrm{TeV}$ proton beam collisions. Brief detector description, operation conditions and performance of the detector at the initial operation period are presented.

The XIXth International Workshop on High Energy Physics and Quantum Field Theory - QFTHEP2010 Golitsyno, Moscow, Russia

September 08-15 2010

Speaker 


\section{Introduction}

The ATLAS detector is one of the two general-purpose detectors at the Large Hadron Collider (LHC) [1]. The ATLAS collaboration includes 3000 scientists from 147 institutes and 38 countries. This detector was fully completed in the underground cavern at CERN in August 2008. Two years of ATLAS commissioning and operation with cosmic rays preceded the LHC start at the end of 2009. ATLAS recorded experimental data for pp-collisions at the centre-of-mass energies $0.9 \mathrm{TeV}$ and 2.36 $\mathrm{TeV}$ before the end of 2009 with integrated luminosities of approximately $9 \mu \mathrm{b}^{-1}$ [2] and $0.7 \mu \mathrm{b}^{-1}$, respectively. These data sets do not contain very many high- $\mathrm{p}_{\mathrm{T}}$ objects, and therefore do not correspond to the environment for which ATLAS was designed.

Since March 30 up to the beginning of November 2010, the ATLAS experiment operated with proton collisions at the centre-of-mass energy $7 \mathrm{TeV}$. The data, collected before September 5, corresponded to the integrated luminosity $3.46 \mathrm{pb}^{-1}$.

This paper is based mostly on the results of summer 2010 with proton collisions at the energy $7 \mathrm{TeV}$. It includes the brief description of the ATLAS detector, operation conditions at the initial stage of $7 \mathrm{TeV}$ data collection and performance characteristics of the detector.

The ATLAS collaboration has published the first paper with experimental results on March 15, 2010 for charged particle multiplicities and transverse momentum measurements in pp-collisions at $0.9 \mathrm{TeV}$ [2].

\section{ATLAS detector}

The ATLAS detector [1] has $44 \mathrm{~m}$ length, $25 \mathrm{~m}$ diameter and weighs $\sim 7000$ tons. It covers almost the entire solid angle around the nominal interaction point and includes the following sub-components: inner detector, muon spectrometer, calorimeter system and specialized detectors in the forward region. The overall ATLAS detector layout is shown in Figure 1.

The inner detector operates inside the axial magnetic field of $2 \mathrm{~T}$ with the function of inner tracking system based on three types of tracking devices. These are the innermost silicon pixel detector (Pixel), the silicon strip detector (SemiConducor Tracker, SCT) and the outer tracking with proportional straw tubes with electron identification capability based on transition radiation measurements (Transition Radiation Tracker, TRT). The Pixel contains 3 barrel layers and 2x3 disks in end-caps. SCT has 4 barrel layers and 2x9 discs in end-caps with pairs of single sided sensors. The TRT includes 96 modules in barrel and 112 modules in end-caps with 372032 straws. The inner detector covers pseudorapidity range $|\eta|<2.5(|\eta|<2.0$ for TRT).

The ATLAS detector is optimized for muon identification, with an efficiency greater than $95 \%$ and a fractional momentum resolution better than $3 \%$ over a wide transverse momentum $\left(p_{T}\right)$ range. The muon spectrometer consists of air-core toroids with gas-based muon chambers. It covers the pseudorapidity range $|\eta|<2.7$ for trigger and provides muon momentum measurements for $|\eta|<2.4$. For the muon transverse momentum $\sim 1 \mathrm{TeV}$ resolution is better that $10 \%$. The toroidal magnetic field is $\sim 0.5 \mathrm{~T}$ 
(1 T) on average in the barrel (end-caps) of this spectrometer, resulting in a bending power $2-7.5 \mathrm{Tm}$ for transverse momentum measurements. Over most of the $\eta$-range, tracks are measured by Monitored Drift Tubes (MDT); in the high $\eta$-regime the closest of four wheels to the interaction region is instrumented with Cathode Strip Chambers (CSC). Thin Gap Chambers (TGC) provide trigger information in the end-caps and Resistive Plate Chambers (RPC) in the barrel.

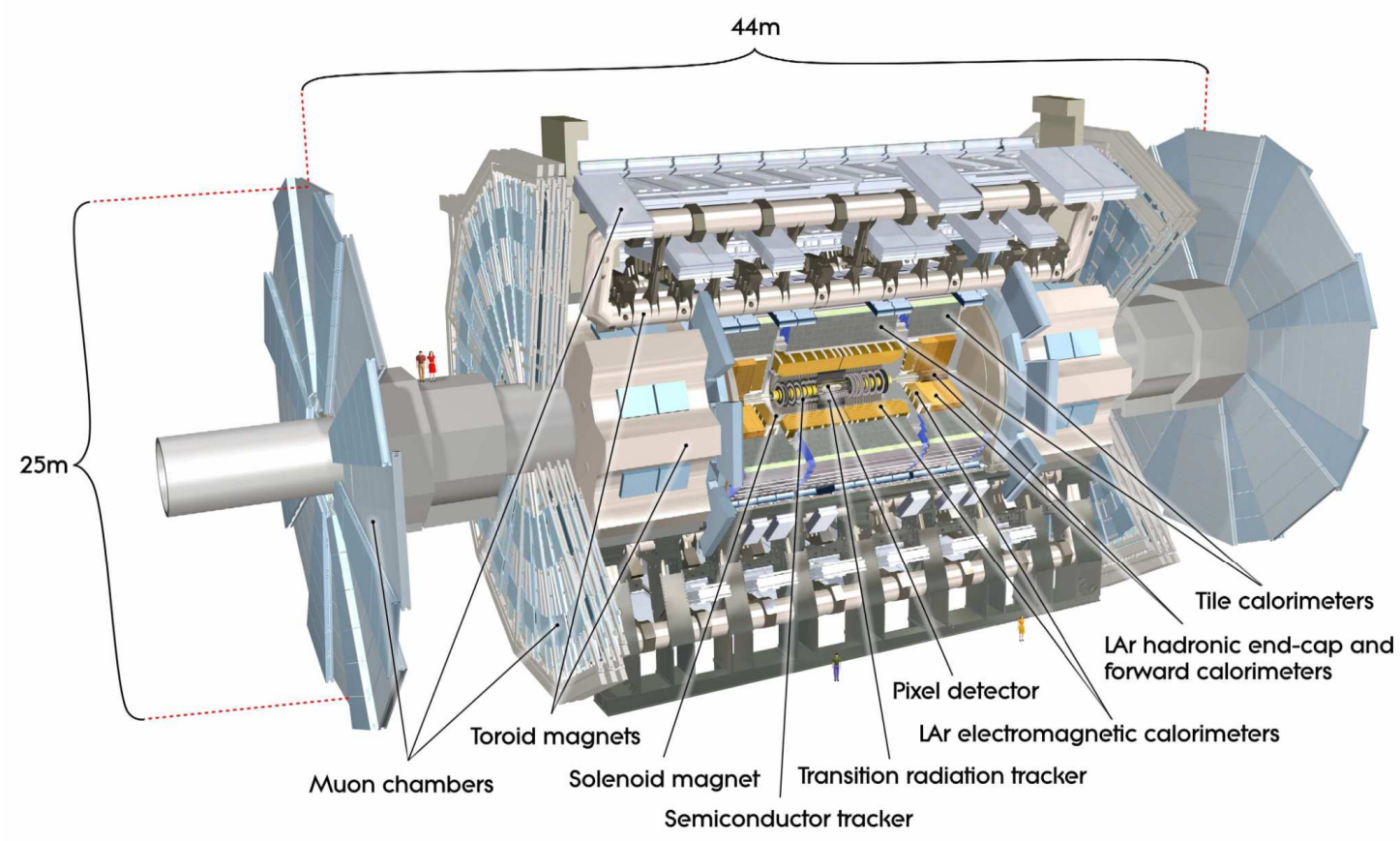

Fig. 1. Cut-away view of ATLAS detector.

The hadronic calorimeter system covers the $\eta$-range of $|\eta|<5$ and provides trigger and missing transverse energy $\left(E_{T}{ }^{\text {miss }}\right)$ measurements with energy resolution $\sigma / \mathrm{E}$ $\sim 50 \% \sqrt{\mathrm{E}}$. The central hadronic calorimeter (Tile) is a sampling calorimeter with steel as the absorber material and scintillator as the active medium and covers the region $|\eta|<$ 1.7. Hadronic end-caps (HEC), the electromagnetic (EM) and forward calorimeters (FCal) are based on a liquid argon (LAr) technology with different types of absorber materials. The electromagnetic calorimeter uses an accordion geometry to ensure fast and uniform response. It provides energy measurement with resolution $\sigma / \mathrm{E} \sim 10 \% \sqrt{\mathrm{E}}$, electron/gamma trigger and identification. A presampler detector, to correct for energy losses in the upstream material, is installed in front of the EM calorimeter in the range $|\eta|<1.8$.

The forward region has two dedicated detectors, the LUCID Cherenkov counter and the Zero Degree Calorimeter (ZDC). In addition, the BPTX - the electrostatic beam-pickup which monitors the timing of the beam near ATLAS - and two scintillator wheels (MBTS) were mounted in front of the electromagnetic end-caps to provide trigger signals with minimum bias events. 
The coordinate system of the ATLAS detector has the origin at the nominal interaction point. The positive $x$-axis is defined as pointing from the interaction point to the centre of the LHC ring, the positive $y$-axis is defined as pointing upwards, and the beam direction defines the $z$-axis of a right-handed coordinate system. Transverse momenta are measured in the $x-y$ plane with radius $r$. Polar $(\theta)$ and azimuthal $(\varphi)$ angles are measured with respect to this reference frame. The pseudorapidity is defined as $\eta=-\ln \tan (\theta / 2)$.

The luminosity weighting relative fraction of detector uptime and good-quality data delivery of the various ATLAS subsystems with stable beams at $\sqrt{s}=7 \mathrm{TeV}$ are shown in Table 1. It corresponds to time period of current run before August 30, 2010. Not included in the numbers is the $1.5 \%$ average DAQ inefficiency during runs with stable beams. Also not included are $36 \mathrm{nb}^{-1}$ that were used for special detector commissioning studies and are not used for physics analyses. The overall data taking efficiency is $93.9 \%$.

Table 1. Luminosity-weighted fraction of the time during stable beam operation for which different detectors were able to take data under nominal conditions.

\begin{tabular}{|c|c|c|c|c|c|c|c|c|c|c|}
\hline \multicolumn{3}{|c|}{$\begin{array}{l}\text { Inner Tracking } \\
\text { Detectors }\end{array}$} & \multicolumn{4}{|c|}{ Calorimeters } & \multicolumn{4}{|c|}{ Muon Detectors } \\
\hline Pixel & SCT & TRT & $\begin{array}{l}\text { LAr } \\
\text { EM }\end{array}$ & $\begin{array}{l}\text { LAr } \\
\text { HAD }\end{array}$ & $\begin{array}{l}\text { LAr } \\
\text { FWD }\end{array}$ & Tile & MDT & RPC & TGC & $\operatorname{CSC}$ \\
\hline 96.7 & 97.5 & 100 & 93.8 & 98.8 & 99.0 & 99.7 & 98.6 & 98.5 & 98.6 & 98.5 \\
\hline
\end{tabular}

\section{ATLAS operation}

The ATLAS data-recorder time profile for proton beam operation in 2010 is shown in Fig.2(left). Cumulative luminosity versus day delivered by LHC (green), and that recorded by ATLAS (yellow) during stable proton beams for $7 \mathrm{TeV}$ centre-of-mass energy. The total integrated luminosity recorded up to September 5, 2010 is $3.46 \mathrm{pb}^{-1}$, the achieved peak luminosity is $1.03 \cdot 10^{31} \mathrm{~cm}^{-2} \mathrm{~s}^{-1}$.

The luminosity is determined from counting rates measured by the luminosity detectors. These detectors have been calibrated with the use of the van-der-Meer beamseparation method, where the two beams are scanned against each other in horizontal and vertical planes to measure their overlap function [4]. The systematic uncertainty of the luminosity measurement is estimated to be $11 \%$, dominated by the uncertainty in the beam current product of $10 \%$. 

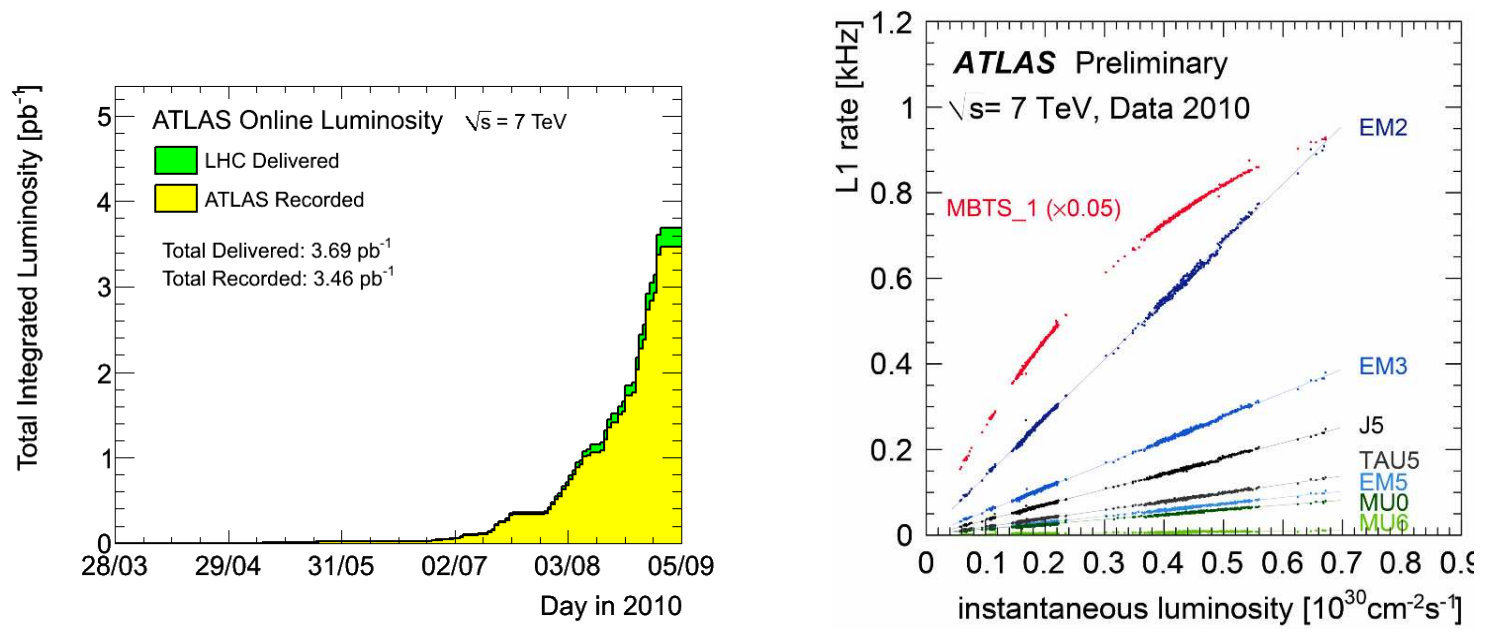

Fig.2. Time profile for integral luminosity with proton $3.5 \mathrm{TeV}$ beams (left). Level 1 trigger rates for instanteneous luminosity up to $0.9 \cdot 10^{30} \mathrm{~cm}^{-2} \mathrm{~s}^{-1}$ (right).

\section{Trigger and event selection}

The ATLAS trigger is composed of three levels of event selection [3]: Level 1 (L1), which is hardware-based, Level 2 (L2), and Event Filter (EF). Level 2 trigger and Event Filter, collectively referred to as the High Level Trigger (HLT), are based on software algorithms analyzing the data on large computing farms. The three levels of the ATLAS trigger system must reduce the output event storage rate to $200 \mathrm{~Hz}$. During the ATLAS startup phase the focus of the trigger selection strategy was to commission the trigger and to ensure that well known Standard Model processes are observed. With peak luminosity less than (few $\times 10^{27}$ ) $\mathrm{cm}^{-2} \mathrm{~s}^{-1}$ the minimum-bias L1 trigger operated using hits in scintillator counters MBTS. HLT operated in pass-through mode, which entails executing the trigger algorithms but accepting the event independent of the algorithmic decision. As the luminosity increased to $>10^{27} \mathrm{~cm}^{-2} \mathrm{~s}^{-1}$, only a fraction of MBTS triggered events were recorded with MBTS prescaled. It is demonstrated in Figure 2(right). Electromagnetic cluster, jet, tau and muon L1 triggers with the lowest thresholds were activated un-prescaled. At luminosities $\sim 10^{29} \mathrm{~cm}^{-2} \mathrm{~s}^{-1}$, HLT chains have been activated to cope with increasing rate while running with low L1 thresholds. The lowest thresholds L1 jet trigger becomes prescaled.

Efficiency of L1 jet trigger with transverse energy of electromagnetic cluster $\mathrm{E}_{\mathrm{T}}^{\mathrm{EM}}>5 \mathrm{GeV}$ is shown in Fig. 3(left) as a function of the calibrated offline jet $\mathrm{p}_{\mathrm{T}}$ for jet rapidity $|y|<2.8$. The efficiency was extracted using a minimum bias trigger. Anti- $\mathrm{K}_{\mathrm{T}}$ jet algorithm with radius $\mathrm{R}=0.4$ is applied for jet selection. Data are in good agreement with MC simulations with PYTHIA.

L1 muon efficiency as a function of muon transverse momentum $\mathrm{p}_{\mathrm{T}}$, measured in spectrometer (muon "Stand Alone" $\mathrm{p}_{\mathrm{T}}, \mathrm{SA} \mathrm{p}_{\mathrm{T}}$ ), is presented in Fig.3(right) for end-caps with $|\eta|>1.05$. The efficiency is calculated with respect to offline muons reconstructed 
with the muon spectrometer and the inner detector. The L1 Region of Interest (RoI) to offline matching criteria is $\Delta \mathrm{R}<0.5$.
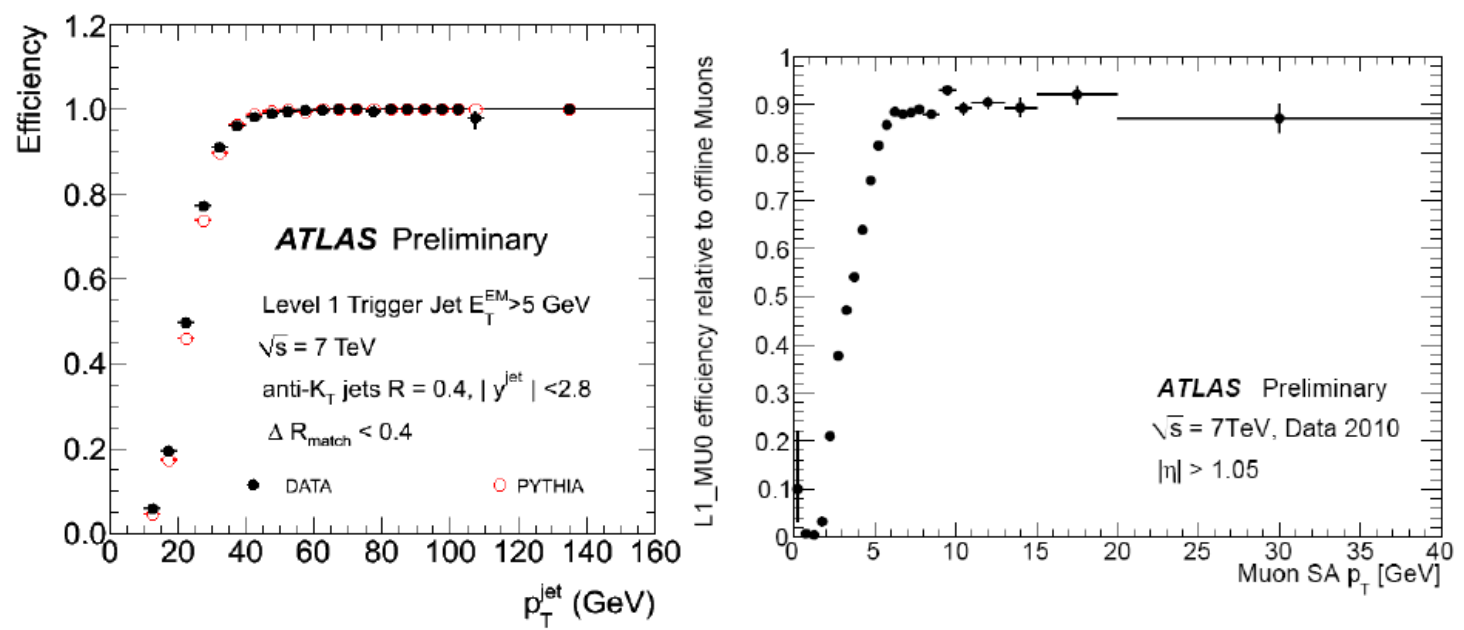

Fig. 3. Level 1 jet trigger efficiency (left); Level 1 muon efficiency for end-caps (right).

To select collision candidates and remove beam-related background, two different strategies were employed [5]:

1) For those studies based mainly on track information, the presence of a primary vertex, reconstructed using at least three tracks with sufficient transverse momenta, typically $\mathrm{p}_{\mathrm{T}}>150 \mathrm{MeV}$, and a transverse distance of closest approach compatible with the nominal interaction point are required. This selection strategy, which was used in [2], uses events triggered by a single hit in one of the two MBTS scintillator wheels.

2) Alternatively, the selection is based on the timing difference of signals detected on both sides of the ATLAS detector. Coincident signals, within a time window of 5 or $10 \mathrm{~ns}$ from either the electromagnetic calorimeters (end-cap or FCal) or from the two MBTS wheels, respectively, are required. The event must again be triggered by an MBTS signal. In case no timing coincidence is found, a two-hit MBTS trigger with at least one hit per side is required.

Without any event selection, the MBTS-triggered events contain backgrounds from beam-related events as shown in Fig. 4(left), where the time difference, $\Delta t_{\text {MBTS }}$, of MBTS signals recorded on both sides of the ATLAS detector is depicted. For events coming from the interaction point $\Delta t_{\text {MBTS }}$ is small. The beam-related background produced upstream or downstream should have $\Delta t_{\text {MBTS }}$ around $25 \mathrm{~ns}$, with the sign giving the direction. Requiring a well-reconstructed vertex with track quality requirements reduces the beam-related background by more than three orders of magnitude while retaining genuine collision events (Fig. 4(right)). 

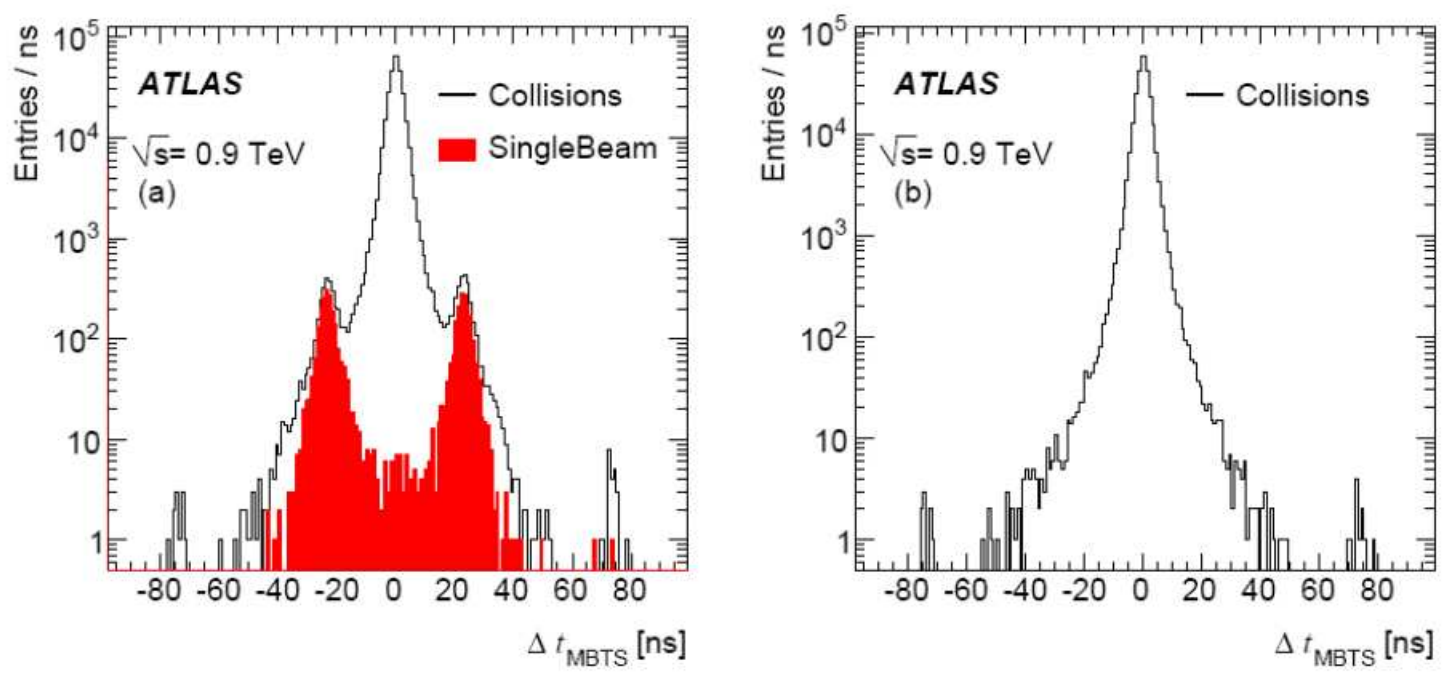

Fig. 4. Time difference $\Delta t$ MBTS of hits recorded by the two MBST scintillator wheels; time difference without any selection (left) and requiring a well-reconstructed vertex (right).

\section{Vertex reconstruction}

The reconstruction of the interaction vertex [5] is based on the reconstruction of charged-particle tracks in the ATLAS inner detector. They are formed from signals in the high-resolution semiconductor pixel and silicon microstrip detectors and, at higher radii with respect to the interaction point, from the information provided by the strawtube tracking detectors. In this analysis, reconstructed tracks fulfilling the following quality requirements are used for the primary vertex reconstruction: $p_{\mathrm{T}}>150 \mathrm{MeV},\left|d_{0}\right|$ $<4 \mathrm{~mm}, \sigma\left(d_{0}\right)<5 \mathrm{~mm}, \sigma\left(z_{0}\right)<10 \mathrm{~mm}$, at least 4 hits in the SCT detector and at least 6 hits in the pixel and SCT detectors. Here $d_{0}$ and $z_{0}$ denote the transverse and longitudinal impact parameters of tracks with respect to the centre of the luminous region, and $\sigma\left(d_{0}\right)$ and $\sigma\left(z_{0}\right)$ denote the corresponding uncertainties as estimated in the track fit. The symbol $p_{\mathrm{T}}$ denotes the reconstructed track transverse momentum. The selection criteria based on the impact parameters are designed to remove a good fraction of the tracks originating from secondary interactions. As estimated from simulations, based on results obtained with the PYTHIA and the full simulation of the ATLAS detector, in non-diffractive $p p$ collisions at $7 \mathrm{TeV}$, the above requirements are fulfilled by $(83.5 \pm 0.1) \%$ of reconstructed tracks corresponding to primary particles.

Two-dimensional distribution of reconstructed primary in the $x$-y plane is presented in Fig. 5(left). In $y$-direction $\sigma(y)=0.040 \mathrm{~mm}$ is larger than in $x$-direction with $\sigma(x)=0.024 \mathrm{~mm}$. Fig. 5(right) shows the dependence of the vertex position resolutions in $z$ on the number of fitted tracks. The resolution is $\sim 0.2 \mathrm{~mm}$ for vertices with 10 fitted tracks. 

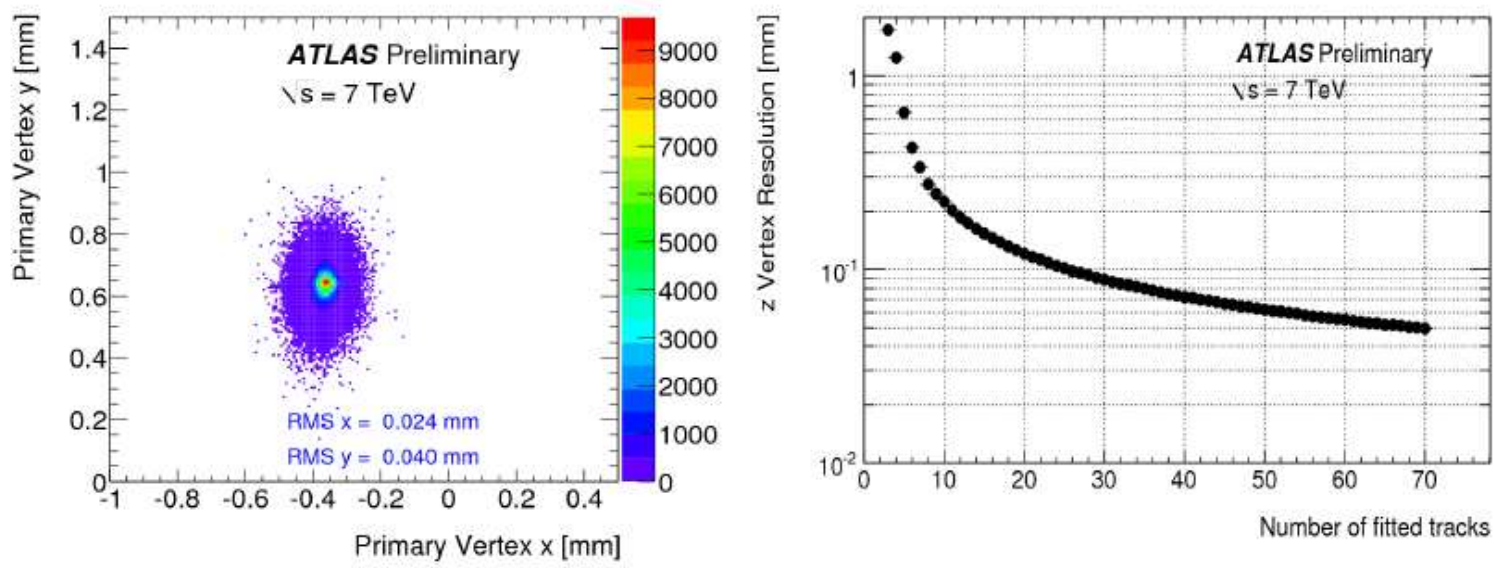

Fig. 5. Two-dimensional distribution of reconstructed primary vertices in the $x-y$ plane (left). Estimated primary vertex $z$ resolution as a function of the number of tracks (right).

The vertex resolution achieved provides a successful reconstruction of events with several primary vertices of pp collisions in the same beam-crossing, as is shown in Fig. 6. The transverse and longitudinal impact parameters of the four vertices are consistent with the luminous region of the colliding beams. The $\mathrm{z}$-coordinates of the vertices are (from left in the plot to right, in $\mathrm{mm}$ ) $-32.1,-22.9,4.6$ and 18.8.

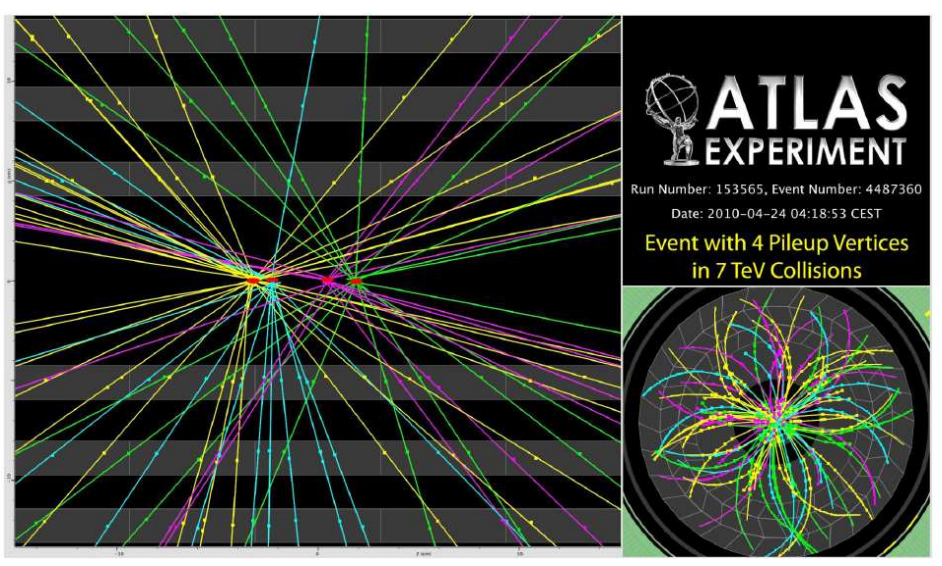

Fig. 6. Event with four primary vertices reconstructed in the same beam-crossing.

\section{Secondary vertices}

The momentum scale and resolution of the tracker, and energy loss within, were all investigated by studying the $\mathrm{K}_{\mathrm{S}}^{0}$ to $\pi^{+} \pi^{-}$decay [6]. The reconstruction requires pairs of oppositely-charged particles compatible with coming from a common vertex. This vertex, in the $\pi \pi$-transverse plane, must be more than $0.2 \mathrm{~mm}$ from the primary vertex. The cosine of the angle between the flight path relative to the primary vertex and the 
momentum vector of the candidate, $\cos \theta_{K}$, is required to exceed 0.8 . The invariant mass distribution, calculated assuming that both charged particles are pions is shown in Fig. 7(left). The simulated signal and background are separately normalized to the data, and the position and width of the $\mathrm{K}_{\mathrm{S}}^{0}$ mass peak are fitted using a Gaussian. The peak in data is at $\mathrm{m}_{\pi+\pi-}=497.5 \pm 0.1 \mathrm{MeV}$, in agreement with the PDG average [7].
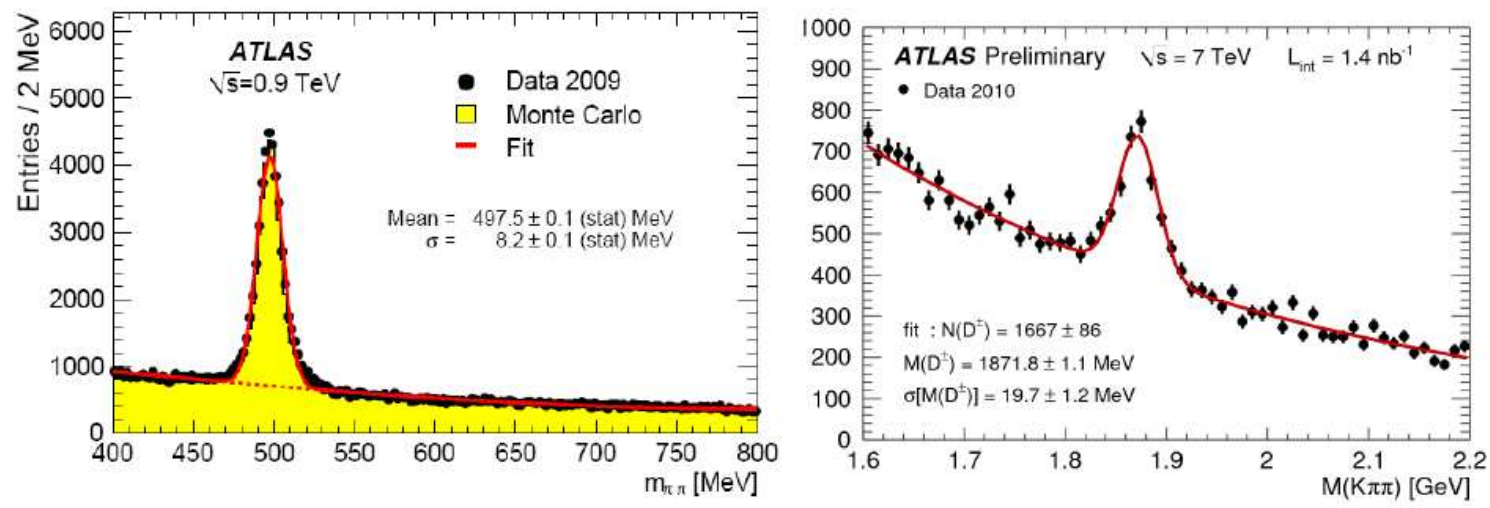

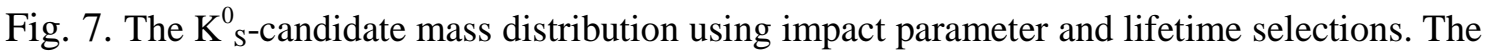
simulated signal and background are separately normalized to the data [6] (left). The $\mathrm{M}\left(\mathrm{K}^{ \pm} \pi \pi\right)$ distribution for the $\mathrm{D}^{ \pm}$- candidates (points). The solid curve represents a fit to the sum of a Gaussian function and an exponential background function (right).

The $D^{ \pm}$- mesons were reconstructed from the decay $D^{+} \rightarrow K^{-} \pi^{+} \pi^{+}[8]$ in pp collisions at $7 \mathrm{TeV}$. In each event, two same-charge tracks, each with $p_{T}>0.8 \mathrm{GeV}$, were combined with a track of the opposite charge and $p_{T}>1.0 \mathrm{GeV}$ to form a $D^{ \pm}$candidate. At least one of the two tracks with the same charge was required to have $p_{T}>$ $1.0 \mathrm{GeV}$. Only three-track combinations successfully fitted to a common vertex with $\chi^{2}$ $<6$ were kept. The $D^{ \pm}$-candidate was required to point back to the primary vertex such that $\left|d_{0}{ }^{P V}\right|<0.15 \mathrm{~mm}$ and $\left|z_{0}{ }^{P V} \sin \theta\right|<0.3 \mathrm{~mm}$. The decay length of the $D^{ \pm}$- candidate was required to satisfy $L_{X Y}>1.3 \mathrm{~mm}$. The pion mass was assigned to the two tracks with the same charge and the kaon mass was assigned to the third track, after which the candidate invariant mass, $M(K \pi \pi)$, was calculated. To suppress the combinatorial background, a cut of $\cos \theta *(K)>-0.8$ was imposed, where $\theta *(K)$ is the angle between the kaon in the $K \pi \pi$ rest frame and the $K \pi \pi$ line of flight in the laboratory frame. To suppress the background from $D^{*^{+}}$-decays, combinations with $M(K \pi \pi)-M(K \pi)<0.15$ $\mathrm{GeV}$ were removed. The background from $D^{+}{ }_{s} \rightarrow \phi \pi^{+}$with $\phi \rightarrow K^{+} K^{-}$was suppressed by rejecting any three-track $D^{+}$-candidate comprised of a pair of oppositely charged tracks which, when assuming the kaon mass for both tracks, has a two-track invariant mass within $\pm 8 \mathrm{MeV}$ of $M(\phi)$. The MC studies estimated that the remaining background from $D^{+} \rightarrow K^{+} K^{-} \pi^{+}$decays was negligible. Fig.7(right) shows the $M(K \pi \pi)$ distribution for the $D^{ \pm}$candidates after all cuts. A clear signal is seen at the nominal value of $D^{+}$mass. The mass distribution was fitted to the sum of a Gaussian function describing the signal and an exponential function describing the non-resonant background. The fitted $D^{ \pm}$yield was $N\left(D^{ \pm}\right)=1667 \pm 86$ events and the fitted mass value, $1871.8 \pm 1.1 \mathrm{MeV}$, was 
consistent with the PDG world average [7]. The width of the signal was $19.7 \pm 1.2 \mathrm{MeV}$ in agreement with the MC expectation.

\section{Electron and photon performance}

With the fine granularity of the EM calorimeter, as well as high-precision tracking and electron identification with the TRT, the ATLAS detector can select and measure effectively electrons, photons and photon conversions. Similar to $\mathrm{K}_{\mathrm{s}}^{0}$ and secondary interaction-vertex measurements, photon conversions are used in material studies of the inner detector. Figure 8(left) shows the distribution of the radialconversion vertices for the inner detector barrel with an event display of a photon conversion in the right part of the figure. The material structure of the inner detector is well reproduced and improvements in the MC material description can be done from these data.

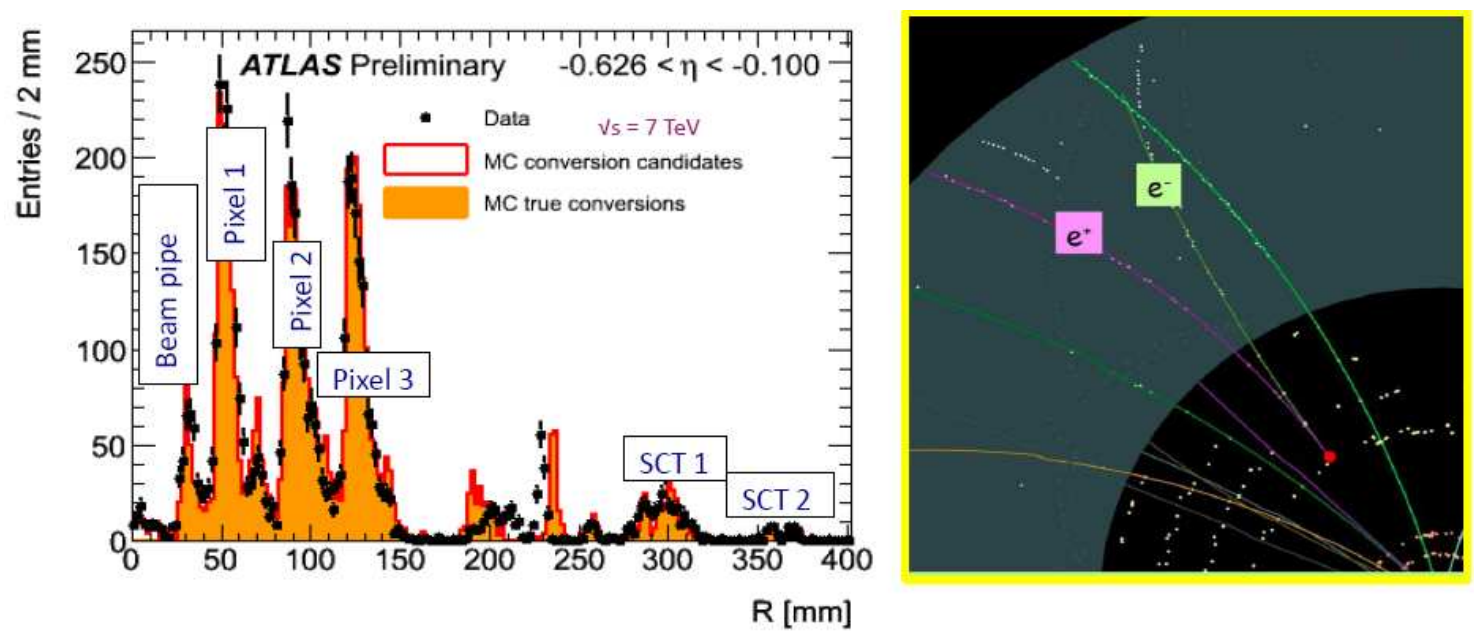

Fig. 8. Radial distribution of the reconstructed conversion vertices in the barrel tracker sections. The three pixel and the first two SCT layers are clearly visible [9](left). Event display of a photon conversion in inner detector at first SCT barrel layer (right).

L1 trigger efficiency (with EM clusters) for photons with the first data is shown in Figure 9(left). The data agree well with minimum bias MC calculations. The measurement results for decays $\pi^{0} \rightarrow \gamma \gamma$ and $\eta \rightarrow \gamma \gamma$ are presented in Figure 9(right). In order to extract $\pi^{0}$ signal the following criteria are applied: 1$)$ well measured photons selected by cluster-removal procedure are used: if two of $3 \times 5$ clusters overlaps, they are both rejected; the acceptance is limited to $|\eta|<2.37$ with excluded transition region between barrel and end-caps $1.37<|\eta|<1.52 ; 2)$ the fraction of energy in the first layer should be larger than 0.1 ; 3) minimun tranverse energy of $3 \times 5$ clusters is $400 \mathrm{MeV}$; 4) only pairs with $\mathrm{p}_{\mathrm{T}}{ }^{\text {pair }}>900 \mathrm{MeV}$ are retained. The number of $\eta \rightarrow \gamma \gamma$ events is expected to be one order of magnitude less than of $\pi^{0} \rightarrow \gamma \gamma$ in the minimum-bias event sample. Therefore, the combinatorial background contribution in the $\eta$-mass region needs to be significantly reduced. This can be achieved by imposing the following kinematic cuts in addition to those of the basic $\pi^{0}$ analysis: transverse cluster energy with $\mathrm{E}_{\mathrm{T}}{ }^{\text {cluster }}>800$ $\mathrm{MeV}$, transverse pair momentum with $\mathrm{p}_{\mathrm{T}}{ }^{\text {pair }}>2200 \mathrm{MeV}$ and no tracks associated with 
the cluster [10]. The diphoton invariant mass spectrum obtained using these cuts is shown in Fig. 9(right) for both data and Monte Carlo. In addition to the $\pi^{0}$ peak, the $\eta \rightarrow \gamma \gamma$ signal can be observed on top of the combinatorial background. The mass spectrum has been fitted using the sum of a Gaussian and a Crystal-Ball function with the same mean value for the $\pi^{0}$-peak, a Gaussian for the $\eta$-peak and a 4 th-order Chebyshev polynomial for the background. The $\pi^{0}$ and background shape parameters are taken from the Monte Carlo simulation while their normalizations are free in the fit, as are the parameters of the Gaussian describing the $\eta$-peak. As can be seen from Fig. 9 (right), the number of data $\eta$-candidates is in agreement with the Monte Carlo simulation. The $\eta$ mass extracted from the fit on data, $m_{\text {Data }}{ }^{\eta}=527 \pm 11$ (stat) $\mathrm{MeV}$, agrees with the expected mass obtained using the same fitting function on the Monte Carlo simulation, $m_{M C}{ }^{\eta}=544 \pm 3$ (stat) $\mathrm{MeV}$, within the statistical and energy scale $(2-3 \%)$ uncertainties. The $1 \%$ difference observed between the Monte Carlo simulation and the nominal PDG value [7] is consistent with the expected accuracy of the specific cluster calibration.
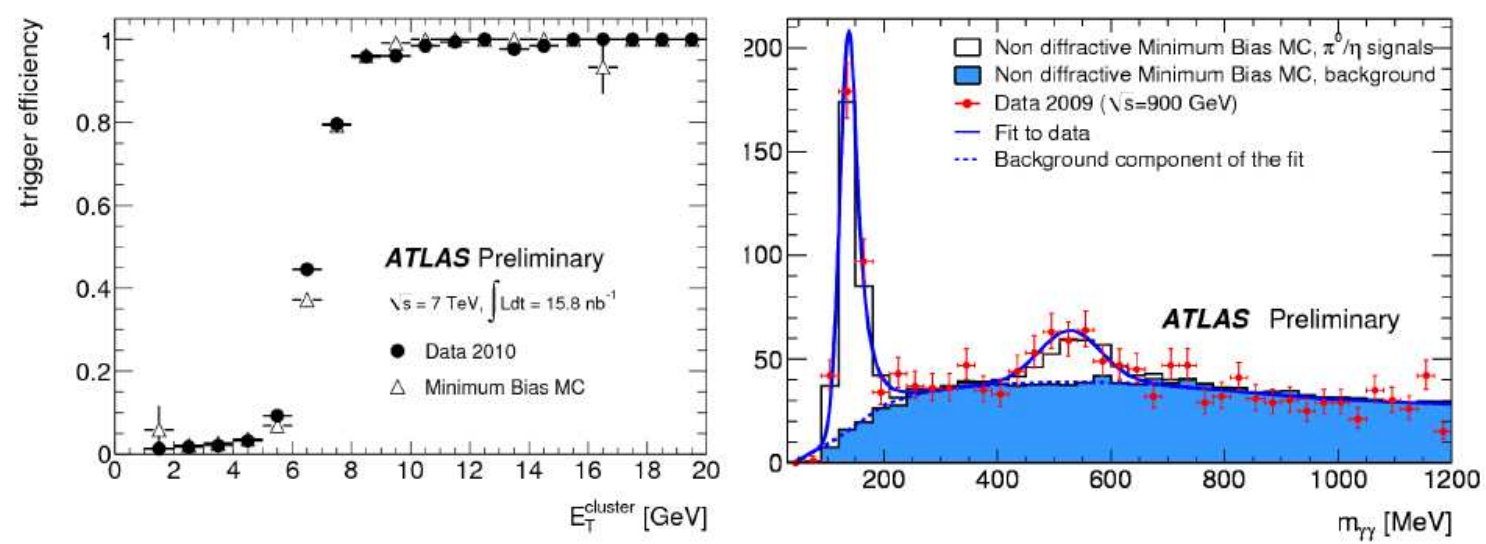

Fig. 9. L1 trigger efficiency for photons (left). Diphoton invariant mass spectrum with selection cuts to extract the $\eta$ peak with the fit superimposed to the data. The Monte Carlo simulation sample is normalized to the number of entries in the data [10] (right).

The baseline electron identification algorithm in ATLAS relies on variables which deliver good separation between isolated electrons and fake signatures from QCD jets. These variables include the information from the calorimeter, the tracker, and the matching between the tracker and the calorimeter. Three reference sets of requirements (loose, medium and tight) have been chosen, providing progressively stronger jet rejection at the expense of some identification efficiency loss. Electron selection starts from the EM calorimeter with the profile of shower development in the layers of the EM calorimeter. Electron reconstruction and identification also rely heavily on tracking performance. High-threshold transition radiation hits from TRT are used for identification of electron tracks associated with EM clusters. The probability to produce high-threshold TRT hits by electrons and pions is measured and results are presented in Fig. 10(left) in dependence on the particle momentum and $\gamma$-factor for TRT barrel. The fraction of high-threshold TRT hits on pion and electron tracks is shown in Fig. 10 
(right) [11]. One can see that with a cut on this parameter, hadron tracks could be well suppressed compare to electrons.
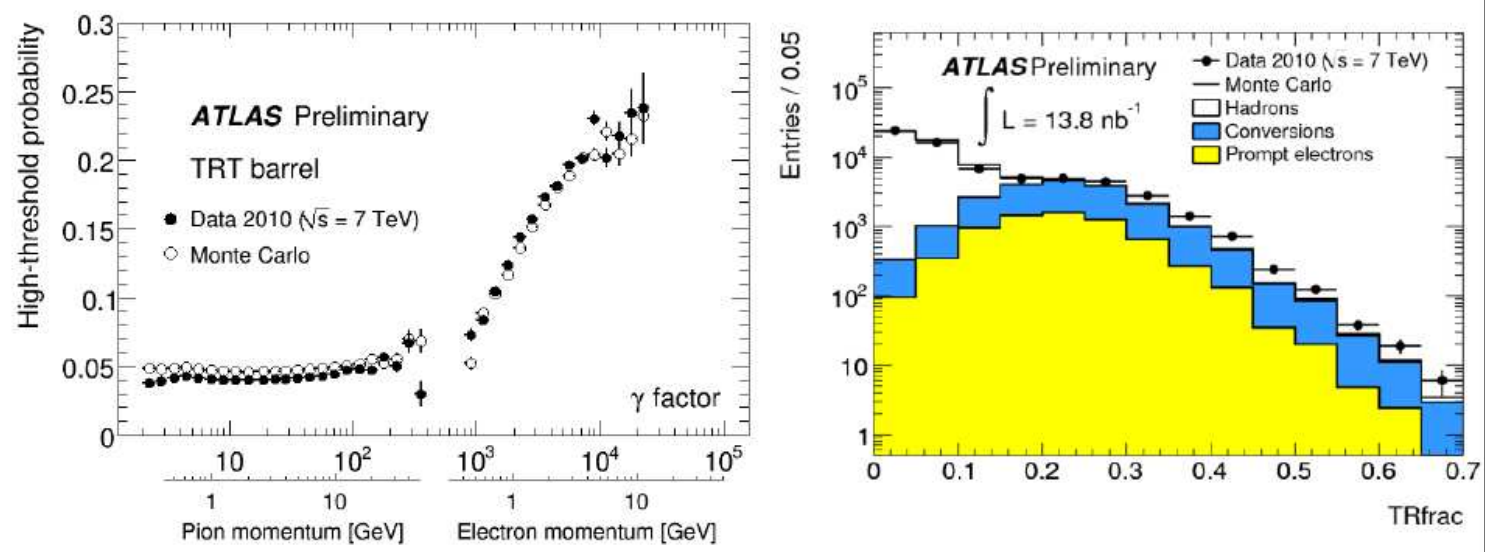

Fig. 10. The probability of high-threshold TRT hits as a function of the Lorentz factor, $\gamma=\mathrm{E} / \mathrm{m}$, and the particle momentum for the TRT barrel (left). The fraction of highthreshold TRT hits on track distribution (TRfrac) [11] (right).

The first observation of inclusive electrons in the collision data collected by the ATLAS at $V_{s}=7 \mathrm{TeV}$, corresponding to a total integrated luminosity of $13.8 \pm 1.5 \mathrm{nb}^{-1}$ is demonstrated in Fig. 11(left) [11]. From a sample of 67124 selected electron candidates with transverse energy above $7 \mathrm{GeV}$, a signal of $9920 \pm 160$ (stat.) \pm 990 (syst.) electrons, predominantly from heavy flavor production (semi-leptonic decays of charm and beauty hadrons), is extracted using a combination of particle identification techniques to determine the dominant hadron and conversion background contributions from data. This signal is compared to expectations from parton shower leading-order Monte Carlo simulations. An important feature of prompt electrons is the presence of a hit in the first pixel layer around beam pipe.
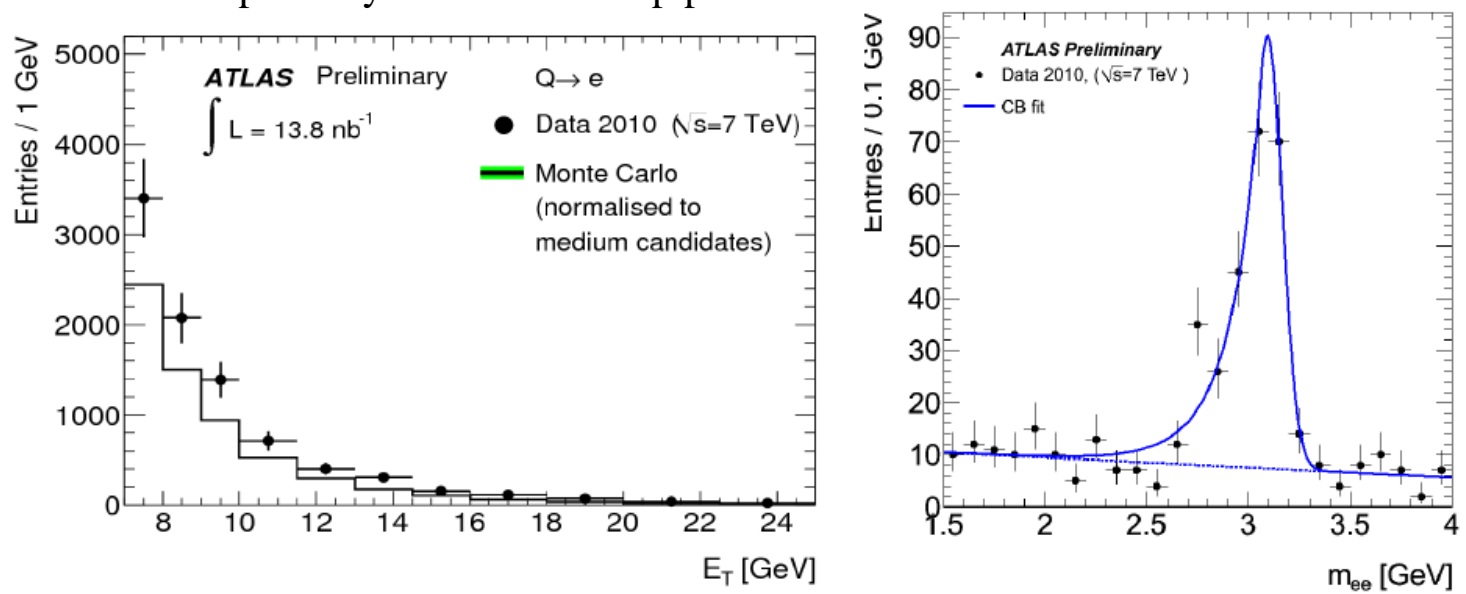

Fig.11. Comparison between data and Monte Carlo of the transverse energy of the prompt electron signal from decays of charm and beauty hadrons, for the medium electron selection. The error bars for the data include statistical and systematic uncertainties and the Monte Carlo prediction has been normalized to the same number of total selected electron candidates as in the data [9] (left). Invariant two electron mass calculated from brem-refit track parameters [12] (right). 
The effective mass of two prompt electron distribution, presented in Fig.11(right), demonstrates a clear signal of the $\mathrm{J} / \psi \rightarrow$ ee process. To extract the signal from the background the following criteria are applied: two electromagnetic clusters matched to tracks with $\mathrm{p}_{\mathrm{T}}>4 \mathrm{GeV}$ for the first electron and $\mathrm{p}_{\mathrm{T}}>2 \mathrm{GeV}$ for the second one, track quality cuts, electromagnetic shower shape requirements and large TRT highthreshold hits fraction on track ( $>0.18$ for the first electron and $>0.15$ for the second). The invariant mass was fitted from track parameters after recovery to Bremsstrahlung [12]. A result of this recovery process is that particles that do not loose a significant amount of energy will have their momentum overestimated. Correction factors for this momentum overestimation have been extracted from simulated electrons, as a function of $\eta$ and $\mathrm{p}_{\mathrm{T}}$. These correction factors $(\sim 4 \%)$ are then applied to the track prior to constructing the invariant mass. The following results are found from the fit: $\mathrm{N}$ (signal) $=222 \pm 11$ events; $\mathrm{N}$ (background) $=28 \pm 2$ events; mass peak $\mathrm{M}=3.09 \pm 0.01 \mathrm{GeV}$; mass resolution $\sigma(\mathrm{M})=0.07 \pm 0.01 \mathrm{GeV}$. For signal, the integration of the fit is from 1.5 to $4 \mathrm{GeV}$, while for background, the integration of the fit result runs from $\mathrm{M}-3 \sigma$ to $\mathrm{M}+2 \sigma$. The mass value found is in agreement with PDG [7].

\section{Muon performance}

The performance of the ATLAS muon reconstruction and identification is studied with $17 \mathrm{nb}^{-1}$ of LHC p-p collision data at $\sqrt{ } s=7 \mathrm{TeV}$ collected with muon triggers [13]. The selected muons have on average a momentum of $11 \mathrm{GeV}$ and a transverse momentum of $4.5 \mathrm{GeV}$ and are predominantly produced in weak decays of light hadrons and of hadrons containing $\mathrm{b}$ and $\mathrm{c}$ quarks. With such single muon events, the redundancy in the ATLAS muon reconstruction allows performance measurements by comparing different identification and reconstruction algorithms.

Muon momenta are independently measured in the inner detector and the muon spectrometer in the range $|\eta|<2.4$. The current estimates of the resolution of the muon momentum measured in the muon spectrometer relative to the inner detector momentum measurement are shown in Fig. 12(left). They are obtained using muons from a fully refitted combination of an inner detector (ID) track and a muon spectrometer (MS) track (combined muons) and are provided as a function of the muon $p_{T}$. These results demonstrate that the resolution with collision data has improved compared to cosmic measurements.

A performance study of $\mathrm{J} / \psi \rightarrow \mu^{+} \mu^{-}$decays is presented from $7 \mathrm{TeV}$ protonproton collisions using the ATLAS detector at the LHC with an integrated luminosity of $78 \mathrm{nb}^{-1}[14]$. The $\mathrm{J} / \psi$ mass was extracted from the reconstructed di-muon invariant mass spectrum using track parameters determined from the inner detector. The agreement with the PDG [7] value of the reconstructed $\mathrm{J} / \psi$ mass is within statistical uncertainties and provides a validation of the momentum scale determination in the low mass region. The mass resolution is compatible with Monte Carlo expectations. The invariant mass distribution for all oppositely-charged muon pairs passing the selection criteria is shown in Fig. 12(right). The $\mathrm{J} / \psi$ mass returned by the fit is $3.095 \pm 0.001 \mathrm{GeV}$, which is consistent with the PDG value $3.096916 \pm 0.000011 \mathrm{GeV}$ [7]. The number of the $\mathrm{J} / \psi$ signal decays is $5350 \pm 90$ and the mass resolution of $\mathrm{J} / \psi$ signal is $71 \pm 1 \mathrm{MeV}$. The 
number of the background pairs in the mass range $2.88-3.31 \mathrm{GeV}$, corresponding to $\mathrm{m}_{\mathrm{J} / \psi} \pm 3 \sigma_{\mathrm{m}}$, is $2560 \pm 60$. In the same figure, the mass distribution obtained from the prompt $\mathrm{J} / \psi$ Monte Carlo sample, normalized to the number of signal events extracted from the fit to data, is also shown.

The $\mathrm{W}$ and $\mathrm{Z}$ bosons are expected to be produced abundantly at the LHC, and for the first time in proton-proton collisions. The ATLAS muon identification and reconstruction algorithms take advantage of the multiple subdetector technologies which provide complementary approaches and cover pseudorapidities up to 2.7 over a wide $p_{T}$ range. The stand-alone muon reconstruction is based entirely on muonspectrometer information, independently of whether or not this muon-spectrometer track is also reconstructed in the inner detector. During first months of ATLAS operation with proton collisions at $\sqrt{\mathrm{s}}_{\mathrm{s}}=7 \mathrm{TeV}$, tens of candidates of $\mathrm{W}$ and $\mathrm{Z}$ decays with high momentum muons and electrons as decay products, were recorded. In Fig. 13 an event with a candidate $\mathrm{Z} \rightarrow \mu \mu$ is presented. Tracks of $\mu^{-}$in barrel and $\mu^{+}$in forward region and corresponding signals in muon chambers in different projections are shown.
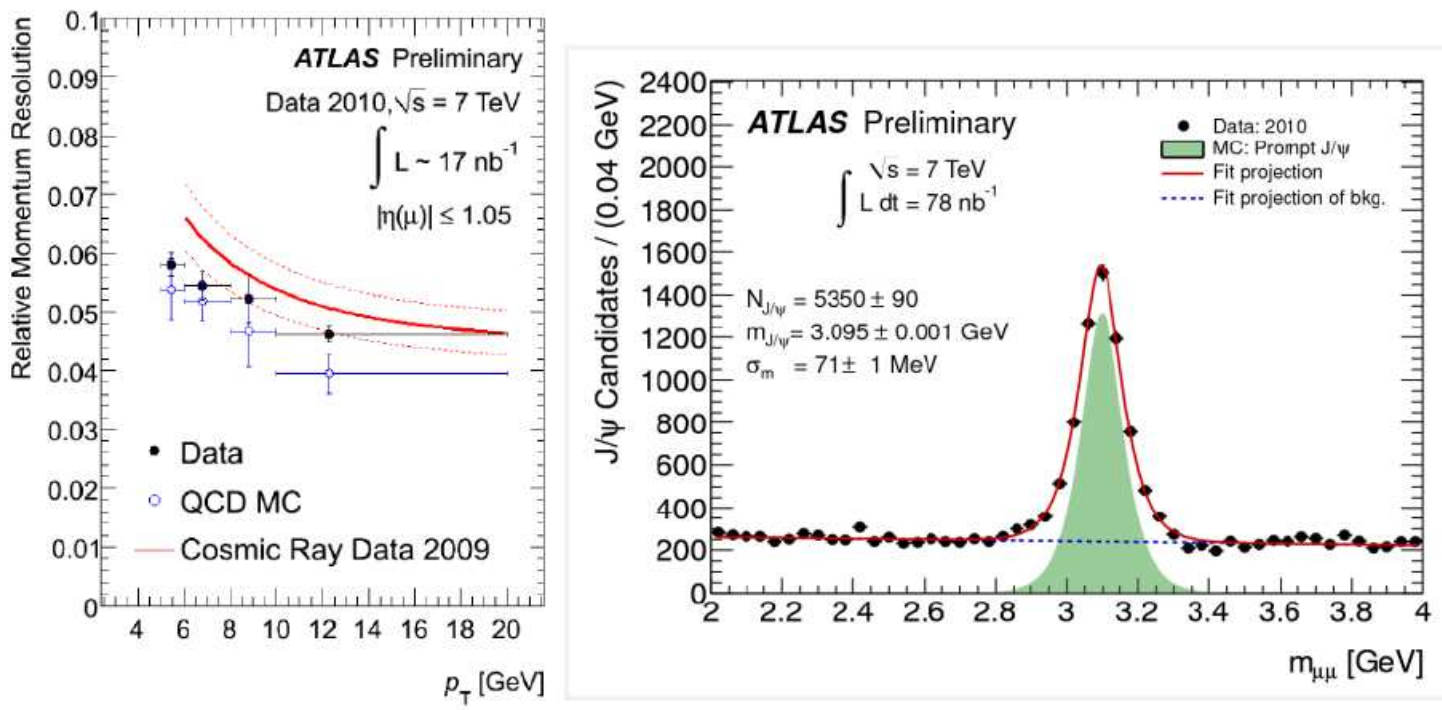

Fig.12. Resolution for the barrel $(|\eta|<1.05)$ as a function of the combined muon $p_{T}$, obtained on collision data from 2010 (black, full dots). Overlaid are shown the expectations from QCD Monte-Carlo (blue). In red the fitted relative resolution of the MS obtained using Cosmic ray data, with an uncertainty band corresponding to $\pm 1 \sigma$ (left). The invariant mass distribution of the reconstructed $\mathrm{J} / \psi \rightarrow \mu \mu$ candidates from data is shown as the black points (right). The solid line is the projection of the result of the unbinned maximum likehood fit to all di-muon pairs in the mass range $2-4 \mathrm{GeV}$, the dashed line is the projection for the background component of the same fit. The filled distribution shows the prompt $\mathrm{J} / \psi$ Monte Carlo predictions, normalized to the number of signal events extracted from the fit to data. 


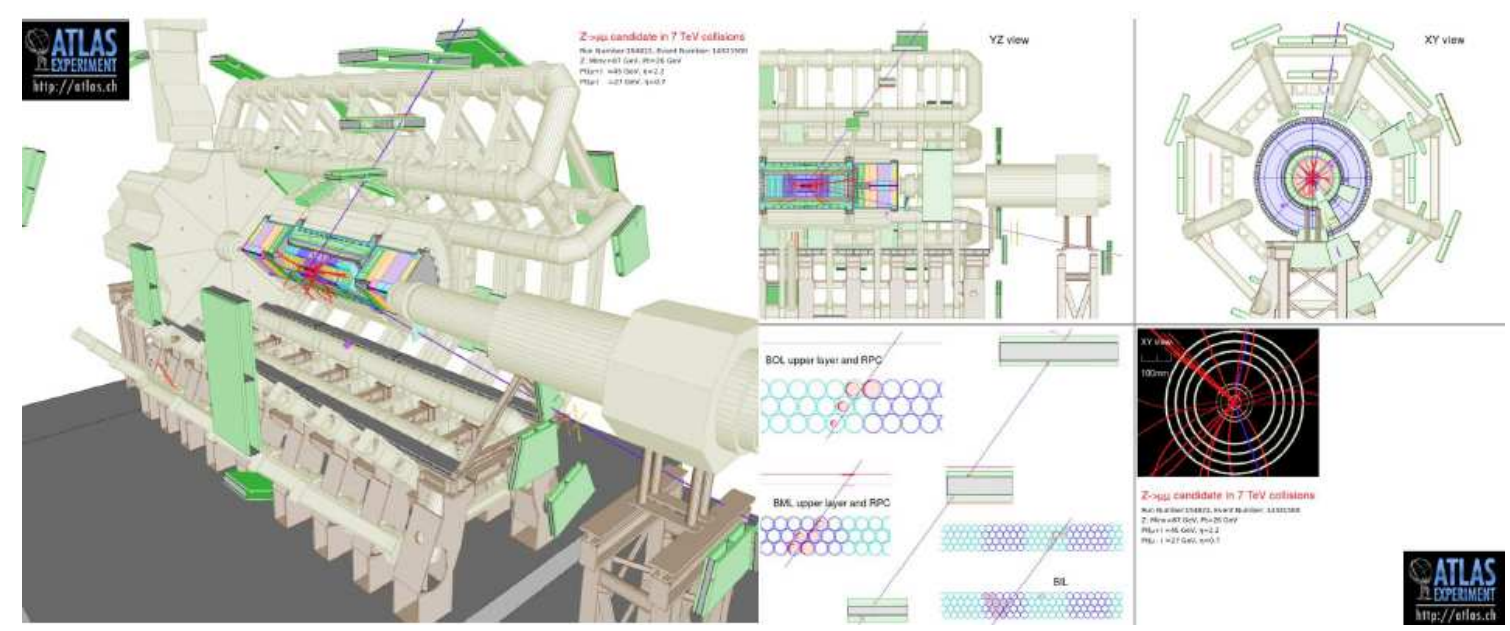

Fig.13. Candidate for $\mathrm{Z} \rightarrow \mu \mu$ decay, collected on 10 May 2010.

Event properties: $\mathrm{p}_{\mathrm{T}}\left(\mu^{+}\right)=45 \mathrm{GeV} ; \eta\left(\mu^{+}\right)=2.2 ; \mathrm{p}_{\mathrm{T}}\left(\mu^{-}\right)=27 \mathrm{GeV} ; \eta\left(\mu^{-}\right)=0.7 ; \mathrm{m}_{\mu \mu}=87 \mathrm{GeV}$

\section{Jets and missing transverse energy performance}

Jets and missing transverse energy $\mathrm{E}_{\mathrm{T}}{ }^{\mathrm{miss}}$ are key objects for physics at colliders. They participate in studies of the Standard Model and beyond. In $\mathrm{E}_{\mathrm{T}}{ }^{\text {miss }}$ measurements all detector subsystems participate, with essential contribution from calorimeters. In addition, jets are identified and their properties studied using tracks measured by the inner detector.

A fist analysis of missing transverse energy with large number of collected proton-proton collision events at a center-of-mass energy of $7 \mathrm{TeV}$ demonstrates good performance of the reconstruction and calibration of the $\mathrm{E}_{\mathrm{T}}{ }^{\text {miss }}$ with up to $300 \mathrm{GeV}$ total transverse energy accumulated per event and with reconstructed objects at large transverse momentum $p_{T}$ [15]. In Fig. 14(left) experimental data for $\mathrm{E}_{\mathrm{T}}{ }^{\text {miss }}$ with 15.2 million minimum-bias events are presented. For $\mathrm{E}_{\mathrm{T}}{ }^{\text {miss }}$ reconstruction the calorimeter energy is used, corrected for the non-compensating calorimeter design and for energy losses in dead material. In addition, the contribution in $\mathrm{E}_{\mathrm{T}}{ }^{\text {miss }}$ coming from muons in the event is studied. The data in Fig. 14(right) demonstrate the performance of $\mathrm{E}_{\mathrm{T}}{ }^{\text {miss }}$ in the presence of high- $\mathrm{p}_{\mathrm{T}}$ objects. The resolution and the tails of the missing transverse energy distribution, calibrated using different schemes, are in good agreement with the simulation. 

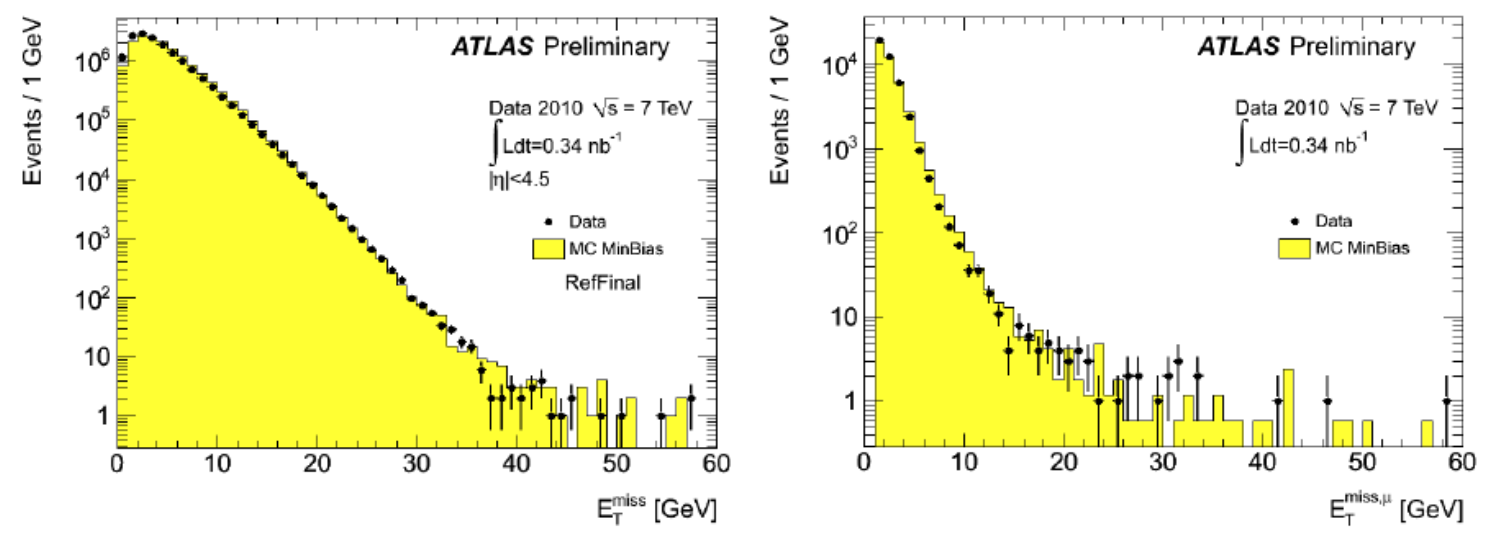

Fig.14. Distribution of $\mathrm{E}_{\mathrm{T}}{ }^{\mathrm{miss}}$ as measured in a data sample of 15.2 million selected minimum bias events (dots) at $7 \mathrm{TeV}$. The expectation from Monte Carlo simulation is superimposed (histogram) and normalized to the number of events in data (left). Distribution of $\mathrm{E}_{\mathrm{T}}{ }^{\mathrm{miss}}$ computed from muons $\left(\mathrm{E}_{\mathrm{T}}{ }^{\text {miss, } \mu}\right)$ in events with at least one reconstructed muon for data (dots) and Monte Carlo simulation (histograms) at $7 \mathrm{TeV}$. The number of events in Monte Carlo simulation is normalized to the number of events in data (right).

The jet cross-sections have been measured with the ATLAS detector for the first time in proton-proton collisions at the centre-of-mass energy of $7 \mathrm{TeV}$, using an integrated luminosity of $17 \mathrm{nb}^{-1}$ [16]. The anti- $\mathrm{k}_{\mathrm{T}}$ algorithm is used to identify jets, using two jet radius parameters, $\mathrm{R}=0.4$ and 0.6 . Inclusive single-jet differential cross sections are presented as a function of jet transverse momentum in Fig.15(left). The results are compared to expectations based on next-to-leading-order QCD, which agree with the data.

Jets are identified and their properties are studied using tracks measured by the ATLAS inner detector [17]. Events are selected using a minimum-bias trigger, allowing the emergence of jets at low transverse momentum to be observed and for jets to be studied independently of the calorimeter. Jets are reconstructed using the anti-kt algorithm applied to tracks with two parameter choices, $\mathrm{R}=0.4$ and 0.6 . The probability that a particular particle carries a fixed fraction of the jet momentum (fragmentation function) is also measured. Fig. 15(right) presents the corrected fragmentation function in anti- $\mathrm{k}_{\mathrm{t}}$ jets with $\mathrm{R}=0.6$ for charged jet $p_{T}$ from $15 \mathrm{GeV}$ to $24 \mathrm{GeV}$, compared with several Pythia 6 tunes, as well as Phojet and Pythia 8. Comparison of the theoretical models implemented in existing tunings of Monte Carlo event generators with the data indicates reasonable overall agreement between data and Monte Carlo.

Jet studies with tracks provide the jet identification and b-tagging, which are important for top quark selection. A few methods exist for b-jet identification, based on track impact parameter measurements, secondary vertex reconstruction and lepton identification. Fig. 16 shows an event in a proton-proton collision at $7 \mathrm{TeV}$ with a reconstructed b-jet in different projections. Four tracks belong to the reconstructed secondary vertex of b-decay inside the beam pipe. 

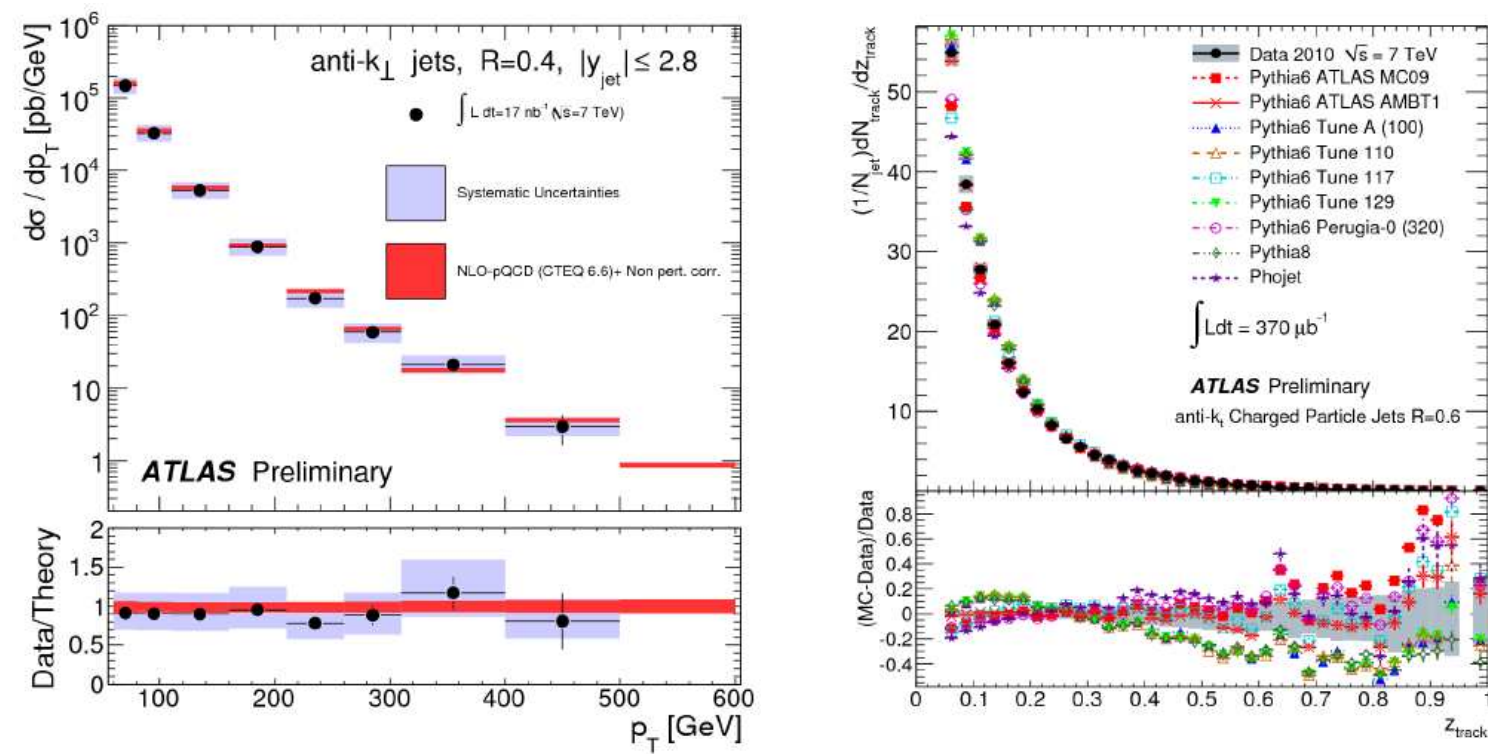

Fig. 15. Inclusive jet differential cross section as a function of jet $p_{T}$ integrated over the region $|y|<2.8$ for jets identified using the anti- $k_{T}$ algorithm with $R=0.4$. The data are compared to NLO QCD calculations to which soft QCD corrections have been applied. The error bars indicate the statistical uncertainty on the measurement, and the grey shaded band indicates the quadratic sum of the systematic uncertainties, dominated by the jet energy scale uncertainty. There is an additional overall uncertainty of $11 \%$ due to the luminosity measurements that is not shown. The theory uncertainty shown in orange is the quadratic sum of uncertainties from the choice of renormalization and factorization scales, parton distribution functions, running constant $\alpha_{\mathrm{s}}\left(M_{\mathrm{Z}}\right)$, and the modeling of soft QCD effects (left).

Corrected fragmentation function in anti- $\mathrm{k}_{\mathrm{t}}$ jets with $\mathrm{R}=0.6$ for charged jet $p_{T}$ from $15 \mathrm{GeV}$ to $24 \mathrm{GeV}$, compared with several Pythia 6 tunes, as well as Phojet and Pythia 8. The shaded area is the total uncertainty for the corrected data distribution (right).

\section{Conclusions.}

The ATLAS detector has demonstrated stable operation and good quality data delivering with the LHC restart at the end of 2009 and during the operation period of 2010 with proton collisions at centre-of-mass energy of $7 \mathrm{TeV}$. Calibration, alignment, synchronization, reconstruction software and trigger on real data have been realized and significant improvements have been achieved. The measurements in the new high energy range show a high-quality performance of the ATLAS experiment. First physics results have been received. High $p_{T}$ objects, such as as jets, leptons, W/Z, b-jets have been observed in a new environment. 


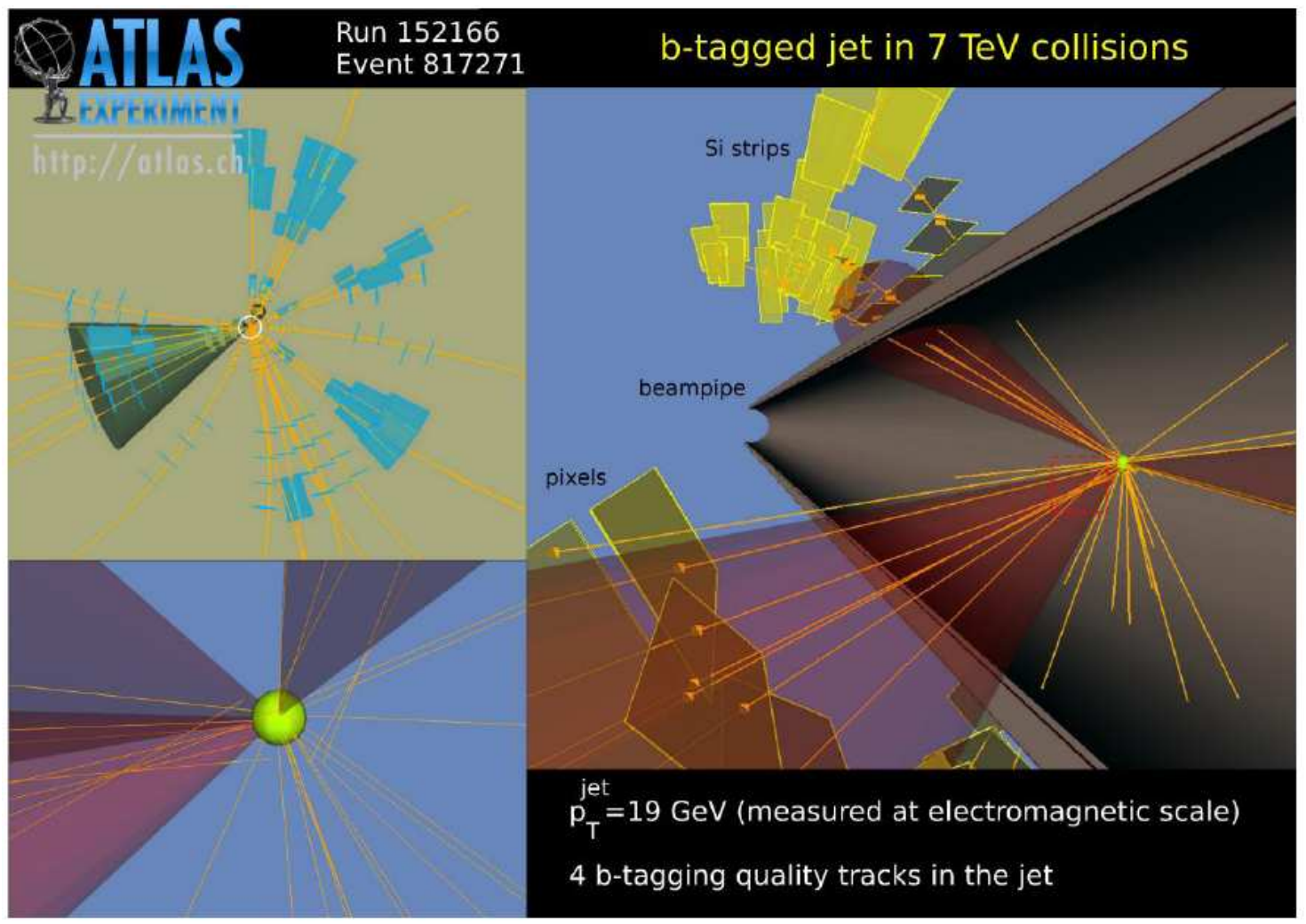

Fig.16. A pp interaction at $7 \mathrm{TeV}$ with reconstructed b-jet candidate with four tracks. Transverse jet momentum $p_{T}$, measured at electromagnetic scale, is $19 \mathrm{GeV}$.

\section{References}

[1] The ATLAS Collaboration, G.Aad et al., The ATLAS Experiment at the CERN Large Hadron Collider, JINST 3 (2008) S08003

[2] The ATLAS Collaboration, G.Aad et al., Charged-particle multiplicities in $p p$ interactions at $\sqrt{ }_{s}=900 \mathrm{GeV}$ measured with the ATLAS detector at the LHC, Phys. Lett. B 688 (2010) 21 [arXiv:1003.3124]

[3] The ATLAS Collaboration, G.Aad et al., Expected Performance of the ATLAS Experiment, Detector, Trigger and Physics, CERN-OPEN-2008-020 (2008).

[4] The ATLAS Collaboration, Luminosity determination using the ATLAS detector, ATLAS-CONF-2010-060.

[5] The ATLAS Collaboration, Performance of primary vertex reconstruction in proton-proton collisions at $\sqrt{s}_{\mathrm{s}}=7 \mathrm{TeV}$ in the ATLAS experiment, ATLAS-CONF2010-069. 
[6] The ATLAS Collaboration, Performance of the ATLAS detector using first collision data, JHEP 1009:056, 2010.

[7] C. Amsler et al., Review of Particle Physics, Phys. Lett. B 667 (2008) 1.

[8] The ATLAS Collaboration, $D^{(*)}$ mesons reconstruction in pp collisions at $\sqrt{ } s=7$ $\mathrm{TeV}$, ATLAS-CONF-2010-034.

[9] The ATLAS Collaboration, Photon conversion plots for Summer conferences 2010, atlas.web.cern.ch/Atlas/GROUPS/PHYSICS/EGAMMA/PublicPlots/20100602.

[10] The ATLAS Collaboration, Performance of the ATLAS electromagnetic calorimeter for $\pi^{0} \rightarrow \gamma \gamma$ and $\eta \rightarrow \gamma$ events, ATLAS-CONF-2010-006.

[11] The ATLAS Collaboration, Observation of inclusive electrons in the ATLAS experiment at $\sqrt{\mathrm{s}}=7 \mathrm{TeV}$, ATLAS-CONF-2010-073.

[12] The ATLAS Collaboration, Electron performance of the ATLAS detector using $J / \psi \rightarrow e^{+} e^{-}$decays,

atlas.web.cern.ch/Atlas/GROUPS/PHYSICS/EGAMMA/PublicPlots/20100721.

[13] The ATLAS Collaboration, Muon Reconstruction Performance, ATLAS-CONF2010-064.

[14] The ATLAS Collaboration, J/ $\psi$ Performance of the ATLAS Inner Detector, ATLAS-CONF-2010-078.

[15] The ATLAS Collaboration, Performance of the Missing Transverse Energy Reconstruction and Calibration in Proton-Proton Collisions at a Center-of-Mass Energy of $7 \mathrm{TeV}$ with the ATLAS Detector, ATLAS-CONF-2010-057.

[16] The ATLAS Collaboration, Measurement of jet production in proton-proton collisions at $7 \mathrm{TeV}$ centre-of-mass energy with the ATLAS Detector, ATLASCONF-2010-050.

[17] The ATLAS Collaboration, Measurement of differential cross section and fragmentation of jets from tracks in proton-proton collisions at center-of-mass energy $\sqrt{\mathrm{s}=7} \mathrm{TeV}$ with the, ATLAS-CONF-2010-049. 